\title{
Comparative Effectiveness of Ruptured Cerebral Aneurysm Therapies: Propensity Score Analysis of Clipping versus Coiling
}

\author{
J.S. McDonald, R.J. McDonald, J. Fan, D.F. Kallmes, G. Lanzino, and H.J. Cloft
}

\begin{abstract}
BACKGROUND AND PURPOSE: The relative merits of treating ruptured aneurysms with clipping versus coiling continue to be a topic of debate. We evaluated a national, multihospital patient data base to examine recent trends in ruptured aneurysm therapies and to compare peri-procedural outcomes between clipping and coiling treatments.
\end{abstract}

MATERIALS AND METHODS: The Premier Perspective data base was used to identify patients hospitalized between 2006-2011 for ruptured aneurysm who underwent clipping or coiling therapy. A propensity score model, representing the probability of receiving clipping, was generated for each patient by use of relevant patient and hospital variables. After Greedy-type matching of the propensity score, the risk of in-hospital mortality and morbidity was compared between clipping and coiling cohorts.

RESULTS: A total of 5229 patients with ruptured aneurysm (1228 clipping, 4001 coiling) treated at 125 hospitals were identified. Clipping therapy frequency decreased from $27 \%$ in 2006 to $21 \%$ in 2011. After propensity score adjustment, in-hospital mortality risk was similar between groups $(\mathrm{OR}=0.94$ [95\% Cl, 0.73-1.21]; $P=.62$ ). However, unfavorable outcomes were more common after clipping compared with coiling, including discharge to long-term care (OR $=1.32[95 \% \mathrm{Cl}, 1.12-1.56] ; P=.0006)$, ischemic complications $(\mathrm{OR}=1.51[95 \% \mathrm{Cl}$, $1.24-1.83] ; P=.0009)$, neurologic complications (OR $=1.64[95 \% \mathrm{Cl}, 1.18-2.27] ; P=.0018)$, and other surgical complications $(\mathrm{OR}=1.55[95 \%$ $\mathrm{Cl}, 1.05-2.33] ; P=.0240)$.

CONCLUSIONS: This study of a data base of multiple hospitals in the United States demonstrates that clipping of ruptured cerebral aneurysms resulted in greater adjusted morbidity compared with coiling.

ABBREVIATIONS: ICD-9 = International Classification of Diseases 9th Revision; ISAT = International Subarachnoid Aneurysm Trial

T he relative merits of treating ruptured aneurysms with clipping versus coiling continue to be a topic of debate. The International Subarachnoid Aneurysm Trial (ISAT) demonstrated a $7.4 \%$ decrease in absolute risk of death or dependency (modified Rankin score, $4-5$ ) at 1 year, with $23.5 \%$ of patients randomly assigned to coiling dependent or dead at 1 year compared with $30.9 \%$ randomly assigned to clipping. ${ }^{1}$ However, because ISAT only enrolled patients who were deemed suitable to receive either clipping or coiling therapy, representing only $22 \%$ of patients who were screened, ${ }^{2}$ the generalizability of the results has been called into question. ${ }^{3}$ Despite this criticism, multiple reports show a trend toward increasing use of coiling over clipping for patients

Received February 13, 2013; accepted after revision April 15

From the Departments of Radiology (J.S.M., R.J.M., D.F.K., G.L., H.J,C.), Health Sciences Research (J.F.), and Neurosurgery (D.F.K., G.L., H.J.C.), Mayo Clinic, Rochester Minnesota.

Please address correspondence to Jennifer S. McDonald, PhD, Department of Radiology Mayo Clinic, 200 1st Street SW, Rochester, MN 55905; e-mail:

mcdonald.jennifer@mayo.edu

http://dx.doi.org/10.3174/ajnr.A3642 with ruptured cerebral aneurysms. ${ }^{4-7}$ Studies of large patient data bases that compared clipping and coiling treatments yielded mixed results, with some demonstrating worse outcomes after clipping $^{8,9}$ and others demonstrating worse outcomes after coiling. ${ }^{6,10}$ In addition, the nonrandomized studies may have been affected by selection bias. To address this disparity in study findings and the concern of potential selection bias, we identified patients hospitalized for ruptured cerebral aneurysm between 2006-2011 by use of a large multihospital data base and performed a propensity score analysis to compare peri-procedural outcomes between patients of similar clinical and demographic characteristics who were treated with clipping or coiling.

\section{MATERIALS AND METHODS Study Population}

The Perspective data base (Premier, Charlotte, North Carolina) is a voluntary, fee-supported collection of data developed to assess the quality and resource utilization of health care delivery within the United States. ${ }^{11}$ As of 2011, the Perspective data base consisted of approximately $15 \%$ of hospitalizations nationwide and repre- 
Table 1: Patient and hospital demographics of patients with ruptured aneurysm

\begin{tabular}{|c|c|c|c|}
\hline & Clipping & Coiling & $P$ Value $^{a}$ \\
\hline No. of patients & 1228 & 4001 & \\
\hline \multicolumn{4}{|l|}{ Patients } \\
\hline Age, y, median (IQR) & $53(45-62)$ & $55(46-65)$ & $<.0001$ \\
\hline Female, $n(\%)$ & $814(66)$ & $2839(71)$ & .0020 \\
\hline \multicolumn{4}{|l|}{ Race, $n(\%)$} \\
\hline White & $742(60)$ & $2290(57)$ & .0512 \\
\hline Black & $193(16)$ & $640(16)$ & .86 \\
\hline Hispanic & $66(5)$ & $220(6)$ & .94 \\
\hline Other & $227(18)$ & $851(21)$ & .0360 \\
\hline \multicolumn{4}{|l|}{ Admission status, $n(\%)$} \\
\hline Elective & $101(8)$ & $480(12)$ & .0002 \\
\hline Urgent & $287(23)$ & $1063(27)$ & .0253 \\
\hline Emergency & $840(68)$ & $2458(61)$ & $<.0001$ \\
\hline \multicolumn{4}{|l|}{ Admission source, $n(\%)$} \\
\hline Nonmedical source & $382(31)$ & $1475(37)$ & .0002 \\
\hline Transfer from hospital/care facility & $350(29)$ & $1350(34)$ & .0006 \\
\hline Emergency department & $470(38)$ & $1106(28)$ & $<.0001$ \\
\hline Clinic & $26(2)$ & $70(2)$ & .40 \\
\hline Charlson score & $1(1-2)$ & $1(1-2)$ & .0054 \\
\hline \multicolumn{4}{|l|}{ Endovascular procedure } \\
\hline Day of procedure (range) & $2(1-3)$ & $2(1-2)$ & $<.0001$ \\
\hline \multicolumn{4}{|l|}{ Hospital } \\
\hline \multicolumn{4}{|l|}{ Region, $n(\%)$} \\
\hline Midwest & $186(15)$ & 450 (11) & .0004 \\
\hline South & $576(47)$ & $1689(42)$ & .0038 \\
\hline Northeast & $266(22)$ & $1307(33)$ & $<.0001$ \\
\hline West & $200(16)$ & $555(14)$ & .0368 \\
\hline No. of beds, $n$ (range) & $623(439-725)$ & $623(448-683)$ & .19 \\
\hline Urban location vs rural, $n(\%)$ & $1215(99)$ & $3908(98)$ & .0051 \\
\hline Teaching vs nonteaching, $n(\%)$ & $818(67)$ & $2721(68)$ & .36 \\
\hline
\end{tabular}

Note:-IQR indicates interquartile range.

a $P$ values derived from Wilcoxon rank sum test or Fisher exact test.

sented more than 600 US hospitals. Detailed information of a patient's hospitalization, including patient demographics, hospital information, diagnoses, procedures, discharge status, and all billed items, are recorded. Time of procedures and administration of billed items, tests, and examinations are reported in relation to the day of admission.

International Classification of Diseases, 9th Revision, Clinical Modification (ICD-9) codes were used to identify all cases of ruptured aneurysm hospitalizations (ICD-9 diagnostic code 430) recorded from 2006-2011. Patients were included if they underwent aneurysmal clipping (ICD-9 procedural code 39.51, "clipping of aneurysm") or coiling (ICD-9 procedural codes 39.52, "other repair of aneurysm"; 39.72, "endovascular repair of occlusion of head and neck vessels"; 39.75 , "endovascular embolization or occlusion of vessel[s] of head or neck by use of bare coils"; 39.76, "endovascular embolization or occlusion of vessel[s] of head or neck by using bioactive coils"; or 39.79, "other endovascular repair [of aneurysm] of other vessels") during hospitalization. Because these diagnostic codes may not be specific for clipping or coiling, billing information was used as confirmation. Patients were included in the clipping or coiling groups if their billing record included an aneurysm clip or endovascular coil(s), respectively.

\section{Outcome Variables}

The primary outcome variables of this study were death during hospitalization, discharge to long-term care (hospice, skilled nursing facility, long-term care hospital, or rehabilitation facil- ity), and ischemic complications (aphasia, ICD-9 diagnostic code 784.3; hemiplegia or paraplegia, 342.0-342.9; or cerebral artery occlusion, 434.0-434.9). Secondary outcomes were defined as hydrocephalus (331.3-331.4), postoperative neurologic complications (997.0-997.09), other postoperative surgical complications (997.2997.5, 998.59, 998.0), ventriculostomy (ICD-9 procedural code 02.2), ventriculoperitoneal shunt surgery (02.34), or tracheostomy (31.3-31.29) that occurred after clipping or coiling. Primary and secondary outcomes defined by ICD-9 codes were excluded from the analysis if they were listed as "present on admission" to specifically examine complications that occurred during hospitalization.

\section{Statistics}

Data were extracted from the Perspective data base by use of SAS (version 9.3; SAS Institute, Cary, North Carolina) and analyzed by use of JMP (version 9, SAS Institute) and R (version 2.15, http:// www.r-project.org/). Continuous results are presented as median and interquartile range to account for nonparametric data distributions. Categoric results are presented as relative frequencies (\%). Patient, procedure, and hospital covariates and outcome incidences were compared between clipping and coiling groups by use of the Wilcoxon rank sum test for continuous variables and Fisher exact test for categoric variables. Propensity score adjustment by use of 1:1 matching methods was performed by use of the MatchIt package in $\mathrm{R}^{12}$

\section{Propensity Score Analysis}

Propensity score analysis was performed as previously described. ${ }^{13}$ Propensity scores, representing the probability of receiving clipping treatment, were calculated for each patient in the clipping and coiling groups by use of a logistic regression model. Twenty-seven covariates were used to generate this propensity score, including patient variables (age, sex, race, admission status, admission source, priority of ruptured aneurysm diagnosis, Charlson comorbidity score [calculated from ICD-9 diagnostic codes as previously described ${ }^{14}$ ], and payor source), clipping/ coiling procedure variables (priority of procedure and day of procedure), and hospital variables (region, number of beds, urban or rural location, and teaching or nonteaching status). After propensity score generation, patients treated with clipping and those treated with coiling underwent 1:1 nearest-neighbor (Greedytype) matching of the logit of the propensity score with a caliper width of 0.25 of the standard deviation of the score. Matching was performed without replacement, and both treated and control units not meeting matching criteria were excluded. Each propensity score-derived matched pair was assigned a unique pair ID by use of an R script. Improvement in covariate balance after match- 


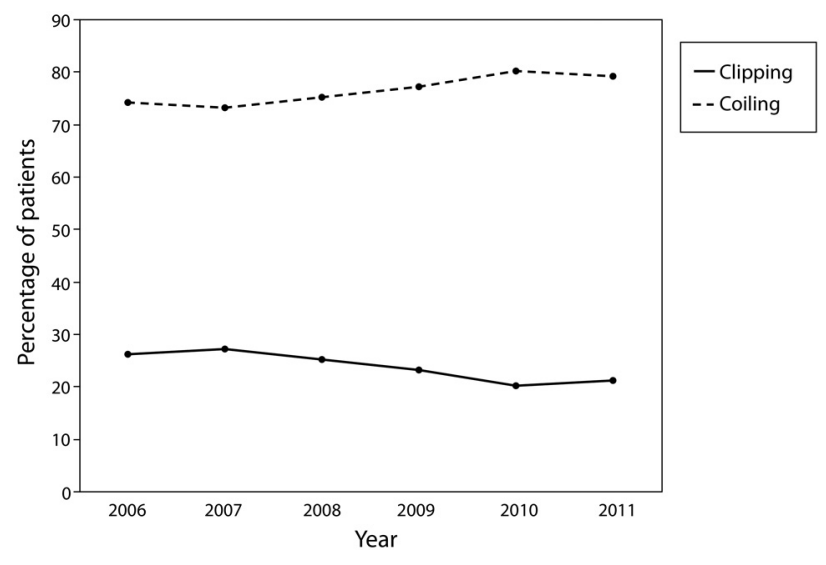

FIG 1. Trends in use of clipping and coiling of ruptured aneurysms from 2006-2011.

ing was analyzed by means of conditional logistic regression, conditioned on the pair ID. Odds ratios of primary and secondary outcomes were calculated after matching by means of the Fisher exact test.

\section{Sensitivity Analysis}

Sensitivity analysis was performed as described by Lin et $\mathrm{al}^{15}$ to assess whether observed differences in outcomes between clipping and coiling groups could be completely attributed to an unmeasured confounder. The lower $95 \%$ confidence interval of the odds ratio of the primary outcome that was the closest to 1.00 when comparing of clipping and coiling groups was used for this analysis. Discharge to long-term care was chosen for sensitivity analysis because the lower 95\% confidence interval (1.12) was closest to equivalency of all primary outcomes significantly different between clipping and coiling groups.

\section{RESULTS}

\section{Patient Demographics}

Between 2006-2011, a total of 5229 patients from 125 unique medical centers were hospitalized with ruptured aneurysms. Within this cohort, 1228 (23\%) patients underwent clipping and 4001 patients $(77 \%)$ underwent coiling (Table 1). Significant differences between patients treated with clipping and those treated with coiling were observed for most patient and hospital variables. Specifically, patients who underwent clipping were younger (median age of 53 versus 55 years, $P<.0001$ ), more likely to have an emergency admission (68\% versus $61 \%, P<$ $.0001)$, and were more likely to be admitted from the emergency department $(38 \%$ versus $28 \%, P<.0001$ ) compared with patients who underwent coiling. Most hospitals that performed clipping or coiling were urban (99\% clipping and $98 \%$ coiling) and classified as teaching hospitals (67\% clipping and $68 \%$ coiling).

\section{Temporal Trends in Clipping versus Coiling}

Trends in the use of clipping and coiling were examined from 2006-2011. The proportion of patients who underwent clipping decreased from $27 \%$ in 2006 to $21 \%$ in 2011 (Fig 1).

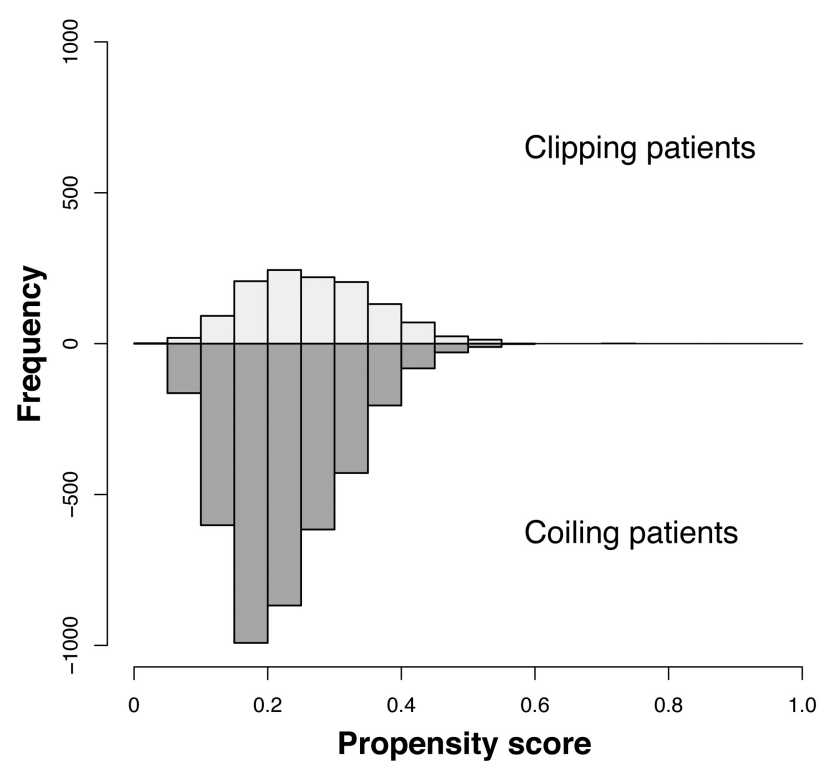

FIG 2. Distribution of propensity scores in the study population. Patients who underwent clipping are shown above the $x$-axis; patients who underwent coiling are shown below the $x$-axis.

\section{Propensity Score-Adjusted Characteristics}

The distribution of unmatched propensity scores for the clipping and coiling groups is shown in Fig 2. Propensity score distributions were very similar between clipping and coiling groups. After 1:1 matching, 1227 patients treated with clipping and 1227 treated with coiling were matched on the basis of similarities in their demographic and clinical characteristics (Table 2). After matching, all covariates were statistically indistinguishable between the clipping and coiling groups.

\section{Propensity Score-Adjusted Outcomes}

Propensity score-adjusted outcome incidence rates and odds ratios are shown in Table 3. After 1:1 matching, the likelihood of in-hospital mortality was similar between the matched clipping and coiling groups (Table 3; OR $=0.94$ [95\% CI, 0.73-1.21], $P=$ .62). There was, however, significantly more morbidity in patients treated with clipping compared with those treated with coiling, as reflected by an increased likelihood of discharge to long-term care $(\mathrm{OR}=1.32$ [95\% CI, 1.12-1.56], $P=.0006)$, ischemic complications $(\mathrm{OR}=1.51$ [95\% CI, 1.24-1.83], $P=.0009)$, postoperative neurologic complications ( $\mathrm{OR}=1.64$ [95\% CI, 1.18-2.27], $P=$ .0018 ), and other postoperative surgical complications ( $\mathrm{OR}=$ 1.55 [95\% CI, 1.05-2.33], $P=.0240$ ). The likelihood of ventriculostomy, ventriculoperitoneal shunt surgery, or tracheostomy was similar between clipping and coiling groups.

\section{Sensitivity Analysis}

The Perspective data base lacks information on cerebral aneurysm size and location. We therefore examined how these and other unmeasured confounders could affect our findings through the use of a sensitivity analysis. This sensitivity analysis (Fig 3) examined the relationship between confounder prevalence in the clipping and coiling groups and the odds ratio of the confounder with respect to discharge to long-term care. For example, if the prevalence of an unmeasured confounder in the clipping group was 
Table 2: Patient and hospital demographics of patients matched by propensity score

\begin{tabular}{|c|c|c|c|}
\hline & Clipping & Coiling & $P$ Value $^{\mathrm{a}}$ \\
\hline No. of patients & 1227 & 1227 & \\
\hline \multicolumn{4}{|l|}{ Patients } \\
\hline Age, y, median (IQR) & $53(45-62)$ & $53(45-63)$ & .19 \\
\hline Female, $n(\%)$ & $814(66)$ & $798(65)$ & .49 \\
\hline \multicolumn{4}{|l|}{ Race, $n(\%)$} \\
\hline White & $741(60)$ & $740(60)$ & .97 \\
\hline Black & $193(16)$ & $196(16)$ & .86 \\
\hline Hispanic & $66(5)$ & $66(5)$ & .99 \\
\hline Other & $227(19)$ & $225(18)$ & .92 \\
\hline \multicolumn{4}{|l|}{ Admission status, $n(\%)$} \\
\hline Elective & $101(8)$ & $100(8)$ & .94 \\
\hline Urgent & $287(23)$ & $309(25)$ & .27 \\
\hline Emergency & $839(68)$ & $818(67)$ & .31 \\
\hline \multicolumn{4}{|l|}{ Admission source, $n(\%)$} \\
\hline Nonmedical source & $382(30)$ & $368(31)$ & .52 \\
\hline Transfer from hospital/care facility & $350(29)$ & $370(30)$ & .35 \\
\hline Emergency department & $469(38)$ & $458(37)$ & .59 \\
\hline Clinic & $26(2)$ & $31(3)$ & .50 \\
\hline Charlson score & $1(1-2)$ & $1(1-2)$ & .34 \\
\hline \multicolumn{4}{|l|}{ Endovascular procedure } \\
\hline Day of procedure (range) & $2(1-3)$ & $2(1-2)$ & .99 \\
\hline \multicolumn{4}{|l|}{ Hospital } \\
\hline \multicolumn{4}{|l|}{ Region, $n(\%)$} \\
\hline Midwest & $189(15)$ & $185(15)$ & .81 \\
\hline South & $576(47)$ & $568(46)$ & .74 \\
\hline Northeast & $266(22)$ & $257(21)$ & .59 \\
\hline West & $200(16)$ & $213(17)$ & .47 \\
\hline No. of beds (range) & $623(439-725)$ & $620(440-683)$ & .85 \\
\hline Urban location vs rural, $n(\%)$ & $1214(99)$ & $1213(99)$ & .83 \\
\hline Teaching vs nonteaching, $n$ (\%) & $817(67)$ & $811(66)$ & .79 \\
\hline
\end{tabular}

Note:-IQR indicates interquartile range.

a $P$ values derived from conditional logistic regression, controlling for paired data through conditional estimation.

$15 \%$ (dashed vertical line) and 5\% in the coiling group (light gray curve), the odds ratio of the confounder would need to be $\sim 2.2$ (point "A") or higher to account for the significant differences observed in discharge to long-term care. If the prevalence of the confounder increased to $10 \%$ in the coiling group, the odds ratio would need to be $\geq 4.3$ (point "B") to account for the observed significant differences in Table 3. Estimates from the Prospective Registry of Subarachnoid Aneurysms Treatment (PRESAT) trial showed that the treatment frequency of large aneurysms was similar between clipping and coiling groups, whereas coiling was preferentially used in posterior circulation aneurysms compared with clipping $(22.5 \%$ versus $4.5 \%) .{ }^{16}$ On the basis of these differences, the confounder odds ratio required to nullify the observed differences in discharge to long-term care approaches infinity because of the asymptotic nature of this function. Given these results, the other measured outcomes would not be affected by treatment disparities in aneurysm size or location.

\section{DISCUSSION}

Our analysis of a large sample of the US population treated for ruptured aneurysm from 2006-2011 found an increasing trend in the proportion of aneurysms treated by coiling versus surgical clipping. After propensity score adjustment, patients treated with coiling and those treated with clipping had a similar likelihood of in-hospital mortality, but patients treated with clipping demonstrated an increased likelihood of morbidity, as defined by discharge to long-term care facilities, ischemic complications, and other postoperative complications, as compared with patients treated with coiling. These results suggest that surgical clipping of ruptured aneurysms generally results in a higher peri-procedural risk of morbidity compared with coiling.

Our findings offer substantial advances over previous corroborating retrospective studies that demonstrated worse outcomes after clipping as compared with coiling. ${ }^{8,9}$ First, we performed propensity score adjustment by use of numerous patient and hospital covariates to minimize the impact of selection bias on the observed treatment outcomes. Second, we performed a sensitivity analysis to measure the effect of a possible unmeasured confounder on our results. Finally, the Perspective data base contains hospitalizations through 2011, enabling an examination of more current clinical practices and patient outcomes compared with these previous studies.

Compared with our study that demonstrated a 5\% lower risk of death or discharge to long-term care in the coiling cohort compared with the clipping cohort, the ISAT investigators found a $10.8 \%$ decrease in absolute risk of death or dependency at 2 months in the coiling cohort compared with the clipping cohort. ${ }^{1}$ The more favorable results from the ISAT study probably can be explained by their exclusion of patients with more guarded initial clinical presentations that probably would put them at higher risk for peri-procedural morbidity and mortality. In contrast, a propensity score-adjusted Canadian study of clipping versus coiling of ruptured intracranial aneurysms treated between 1995-2004 concluded that coiling increased the risk of death or subsequent readmission for SAH (hazard ratio $=1.25$ [95\% CI, 1.00-1.55], $P=.04) .{ }^{6}$ Despite somewhat dissimilar end points, the results of this study led the authors to question the generalizability of the ISAT results. However, the Ontario study was performed at an earlier stage in the development of endovascular coiling compared with ISAT, which may explain the worse outcomes with coiling.

A study of the Nationwide Inpatient Sample by Qureshi et $\mathrm{al}^{5}$ reported different findings in the United States, demonstrating that the in-hospital mortality rates for $\mathrm{SAH}$ admissions decreased from $27-24 \%(P=.003)$ before and after publication of the ISAT results. These observed improvements in outcome probably are a result of multiple factors including shifts in treatment to highervolume centers, ${ }^{17}$ increased operator experience in treating aneurysms with coiling, and technical and procedural improvements that have increased the percentage of aneurysms that are amenable to endovascular coiling. ${ }^{18}$ Additionally, the frequency of coiling increased from $8 \%$ in the 3 years before publication of ISAT to 
Table 3: Patient outcomes after 1:1 matching by propensity score

\begin{tabular}{|c|c|c|c|c|}
\hline & Incidence in Clipping & Incidence in Coiling & $O R^{a}(95 \% \mathrm{Cl})$ & $P$ Value \\
\hline In-hospital mortality & $146 / 1227(12 \%)$ & $154 / 1227(13 \%)$ & $0.94(0.73-1.21)$ & .62 \\
\hline Discharge to long-term care & $520 / 1227(42 \%)$ & $438 / 1227(36 \%)$ & $1.32(1.12-1.56)$ & .0006 \\
\hline Ischemic complications ${ }^{b}$ & $331 / 1227(27 \%)$ & $256 / 1227(21 \%)$ & $1.51(1.24-1.83)$ & .0009 \\
\hline Hydrocephalus & $445 / 1227(36 \%)$ & $500 / 1227(41 \%)$ & $0.83(0.70-0.98)$ & .0210 \\
\hline Postop neuro complications & $109 / 1227(9 \%)$ & $69 / 1227(6 \%)$ & $1.64(1.18-2.27)$ & .0018 \\
\hline Other postop surgical complications & $70 / 1227(6 \%)$ & $46 / 1227(4 \%)$ & $1.55(1.05-2.33)$ & .0240 \\
\hline Ventriculostomy & $462 / 1227(38 \%)$ & $457 / 1227(37 \%)$ & $1.02(0.86-1.20)$ & .84 \\
\hline Ventriculoperitoneal shunt surgery & $148 / 1227(12 \%)$ & $174 / 1227(14 \%)$ & $0.83(0.65-1.06)$ & .11 \\
\hline Tracheostomy & $159 / 1227(13 \%)$ & $157 / 1227$ (13\%) & $1.01(0.80-1.29)$ & .90 \\
\hline
\end{tabular}

Note:-Postop indicates postoperative.

a Odds ratio of clipping versus coiling.

${ }^{b}$ Defined as aphasia, hemiplegia, or paraplegia or cerebral artery occlusion not present at admission.

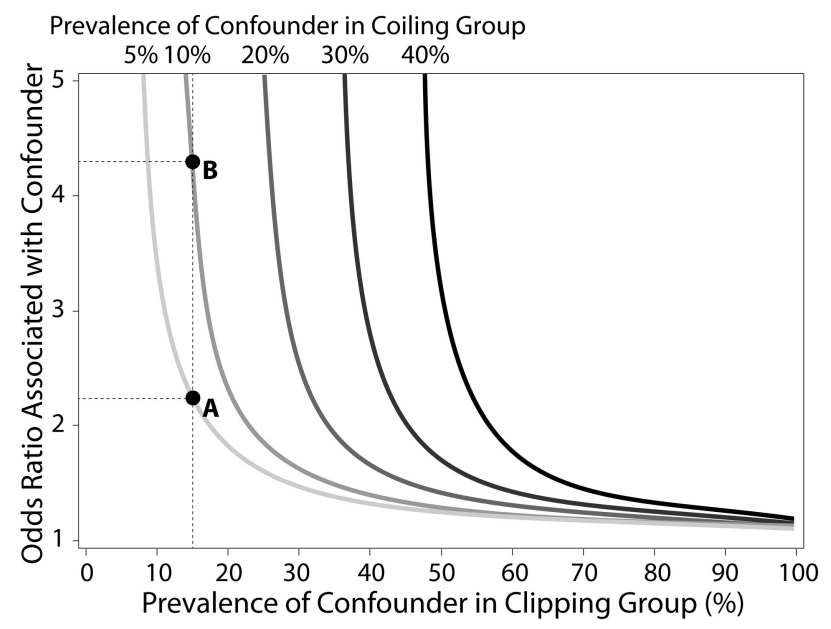

FIG 3. Sensitivity analysis of unmeasured confounders. Sensitivity analysis was performed by use of the lower $95 \%$ confidence interval of the odds ratio of discharge to long-term care (1.12). The $x$-axis represents the hypothetical prevalence of an unmeasured confounder in the clipping group; $y$-axis represents the hypothetical odds ratio associated with this unmeasured confounder. Curved lines represent the required strength of unmeasured confounder (defined by odds ratio[s]) that would be required to nullify the observed differences in discharge to long-term care between treated groups at selected confounder prevalence rates within the coiling group $(5 \%, 10 \%$, $20 \%, 30 \%$, and $40 \%$ ). For a given prevalence within the coiling group, a single unmeasured confounder could potentially nullify significant differences in study outcomes if the data point representing associated odds ratio and clipping prevalence is on or above the depicted curve. Point A depicts an example of a confounder with a prevalence of $15 \%$ in the clipping group and $5 \%$ in the coiling group; in this case, an odds ratio of approximately 2.2 is needed to nullify the significant difference in discharge to long-term care. If the prevalence of the same confounder increased to $10 \%$ in the coiling group, an odds ratio of approximately 4.3 (point B) would be needed to nullify the significant difference.

$43 \%$ in the following 3 years. These results suggest that the incorporation of ISAT results into practice had a favorable impact on mortality. The randomized Barrow Ruptured Aneurysm Trial (BRAT) ${ }^{19}$ provides further evidence to support a shift toward coiling. A poor outcome with death or disability (modified Rankin score $>2$ ) at 1 year occurred in $33.7 \%$ of the patients assigned to clipping and in $23.2 \%$ of the patients assigned to coiling $(P=.02)$. Although most aneurysms randomized to the coil treatment arm in BRAT were treated by coiling, a substantial number crossed over to clipping, which indicates that surgery continues to be an important therapeutic option in many patients.
The increasing proportion of patients with ruptured aneurysm treated with coiling relative to clipping probably reflects how physicians are incorporating the evolving literature into their practice. Despite public debate over the results of the ISAT trial, neurosurgeons are increasingly referring patients with $\mathrm{SAH}$ for endovascular coiling instead of clipping. However, because it is possible that an overly aggressive application of coil embolization could result in a higher complication rate that would offset the benefits of coiling demonstrated in ISAT and BRAT, it is essential that high-quality surgical clipping be available as a complementary treatment option for ruptured cerebral aneurysms. Ideally, ruptured intracranial aneurysms would be treated in centers offering high-quality treatment with open surgical as well as endovascular techniques. The balance between clipping and coiling will vary somewhat between centers, depending on local expertise, and may shift as we learn more about the relative strengths of clipping versus coiling for specific clinical scenarios.

Intracranial aneurysm size and location are important clinical variables used to guide treatment decisions but are not available in the Perspective data base. Because the distributions of aneurysm location and size are expected to differ in the clipping and coiling cohorts, these variables represent potentially important unmeasured confounders. The Hunt and Hess Scale and the Glasgow Coma Scale were also not present in the Perspective data base and also represent potential confounders. If the distribution of these variables were sufficiently disparate between cohorts and were associated with an unfavorable outcome, such an unmeasured confounder could potentially account for the more favorable outcomes observed in the coiling group. Data from the PRESAT trial provide reasonable estimates of the disparities in ruptured aneurysm size and location for patients treated with clipping versus coiling. Given the observed prevalence rates of aneurysm size and distribution in coiling and clipping cohorts, neither variable is a sufficiently powerful unmeasured confounder to nullify the observed differences in discharge to long-term care. However, if other confounders such as a poor Glasgow Coma Scale were present at a substantially higher prevalence in the clipping group (eg, $>4$-fold), they might be able to account for our findings.

Our study has several additional limitations. First, because patients were not randomly assigned to clipping or coiling in our retrospective analysis of the Perspective data base, selection bias may exist. Although propensity score adjustment minimized differences between the clipping and coiling patient groups, other unmeasured variables not contained within the data base, such as 
clinical presentation, may exist and contribute to selection bias. A randomized trial would be necessary to definitively assess the efficacy of treatment of unruptured cerebral aneurysms. Second, coding inaccuracies are known to occur within administrative data bases. ${ }^{20}$ Such inaccuracies, however, are expected to occur randomly with equal prevalence in both clipping and coiling groups. Third, whereas we intentionally excluded complications that were present on admission to only examine complications that occurred during hospitalization, it is possible that some complications were coded incorrectly and occurred independently of clipping or coiling treatment. Finally, long-term outcomes cannot be evaluated in the Perspective data base. However, because the ISAT study demonstrated that 2-month outcomes were reasonably predictive of 1 -year outcomes, ${ }^{21}$ shorter-term outcomes are reasonable surrogate markers for long-term outcomes. Conversely, it might be argued that a higher recurrence rate associated with coiling relative to clipping could result in hemorrhages and/or retreatment procedures that negate some of the improved outcome during initial hospitalization. The results of ISAT, ${ }^{22}$ the CARAT (Cerebral Aneurysm Rerupture After Treatment) ${ }^{23}$ study, and BRAT ${ }^{19}$ demonstrate that it is extremely unlikely that the initial treatment benefit of endovascular coil embolization could be overshadowed by delayed hemorrhages or complications of retreatment.

\section{CONCLUSIONS}

In patients presenting to a large group of US hospitals with ruptured cerebral aneurysms between 2006-2011, surgical clipping was associated with significantly greater morbidity risk than endovascular coiling but with similar in-hospital mortality. Our evaluation of the Perspective data is by no means a randomized study but rather a retrospective look at outcomes on the basis of prevailing practices. Observational data from large clinical data bases can complement the findings of prospective clinical trials because the data base may better reflect real-world practice.

Disclosures: Jennifer S. McDonald-UNRELATED: Grants/Grants Pending: GE Healthcare. ${ }^{*}$ David F. Kallmes_UNRELATED: Consultancy: Medtronic, ${ }^{*}$ ev3, ${ }^{*}$ Codman, ${ }^{*} \mathrm{GE},{ }^{*}$ Comments: Clinical trials planning and implementation, Core Lab services, Cost-effectiveness assessment; Grants/Grants Pending: MicroVention, ${ }^{*}$ Sequent, ${ }^{*}$ Micrus, ${ }^{*}$ Benvenue*; Royalties: UVA Patent Foundation, Comments: Spine Fusion; Travel/Accommodations/Meeting Expenses Unrelated to Activities Listed: MicroVention. * Giuseppe Lanzino—UNRELATED: Consultancy: ev3/Covidien. * Harry J. Cloft_UNRELATED: Grants/Grants Pending: Cordis Endovascular* ( ${ }^{*}$ money paid to institution).

\section{REFERENCES}

1. Molyneux AJ, Kerr RS, Yu LM, et al. International subarachnoid aneurysm trial (ISAT) of neurosurgical clipping versus endovascular coiling in 2143 patients with ruptured intracranial aneurysms: a randomised comparison of effects on survival, dependency, seizures, rebleeding, subgroups, and aneurysm occlusion. Lancet 2005;366:809-17

2. Molyneux A, Kerr R, Stratton I, et al. International subarachnoid aneurysm trial (ISAT) of neurosurgical clipping versus endovascular coiling in 2143 patients with ruptured intracranial aneurysms: a randomised trial. Lancet 2002;360:1267-74

3. Nichols DA, Brown RD Jr, Meyer FB. Coils or clips in subarachnoid haemorrhage? Lancet 2002;360:1262-63

4. Klompenhouwer EG, Dings JT, van Oostenbrugge RJ, et al. Singlecenter experience of surgical and endovascular treatment of rup- tured intracranial aneurysms. AJNR Am J Neuroradiol 2011;32: $570-75$

5. Qureshi AI, Vazquez G, Tariq N, et al. Impact of international subarachnoid aneurysm trial results on treatment of ruptured intracranial aneurysms in the United States. J Neurosurg 2011;114: 834-41

6. O'Kelly CJ, Kulkarni AV, Austin PC, et al. The impact of therapeutic modality on outcomes following repair of ruptured intracranial aneurysms: an administrative data analysis. J Neurosurg 2010; 113:795-801

7. Gnanalingham KK, Apostolopoulos V, Barazi S, et al. The impact of the international subarachnoid aneurysm trial (ISAT) on the management of aneurysmal subarachnoid haemorrhage in a neurosurgical unit in the UK. Clin Neurol Neurosurg 2006;108:117-23

8. Dumont AS, Crowley RW, Monteith SJ, et al. Endovascular treatment or neurosurgical clipping of ruptured intracranial aneurysms: effect on angiographic vasospasm, delayed ischemic neurological deficit, cerebral infarction, and clinical outcome. Stroke 2010;41:2519-24

9. Hoh BL, Chi YY, Lawson MF, et al. Length of stay and total hospital charges of clipping versus coiling for ruptured and unruptured adult cerebral aneurysms in the Nationwide Inpatient Sample database 2002 to 2006. Stroke 2010;41:337-42

10. Cowan JA Jr, Ziewacz J, Dimick JB, et al. Use of endovascular coil embolization and surgical clip occlusion for cerebral artery aneurysms. J Neurosurg 2007;107:530-35

11. Premier Research Services. Premier Perspective Database. Charlotte, North Carolina: 2012

12. Ho DE, Imai K, King G, et al. MatchIt: Nonparametric Preprocessing for Parametric Causal Inference. 2011. http://gking.harvard. edu/matchit. Accessed June 11, 2013.

13. McDonald RJ, McDonald JS, Bida JP, et al. Intravenous contrast induced nephropathy: causal or coincident phenomenon? Radiology 2013;267:106-18

14. Quan H, Sundararajan V, Halfon P, et al. Coding algorithms for defining comorbidities in ICD-9-CM and ICD-10 administrative data. Med Care 2005;43:1130-39

15. Lin DY, Psaty BM, Kronmal RA. Assessing the sensitivity of regression results to unmeasured confounders in observational studies. Biometrics 1998;54:948-63

16. Taki W, Sakai N, Suzuki H. Determinants of poor outcome after aneurysmal subarachnoid hemorrhage when both clipping and coiling are available: Prospective Registry of Subarachnoid Aneurysms Treatment (PRESAT) in Japan. World Neurosurg 2011;76: 437-45

17. Leake CB, Brinjikji W, Kallmes DF, et al. Increasing treatment of ruptured cerebral aneurysms at high-volume centers in the United States. J Neurosurg 2011;115:1179-83

18. Coley S, Sneade M, Clarke A, et al. Cerecyte coil trial: procedural safety and clinical outcomes in patients with ruptured and unruptured intracranial aneurysms. AJNR Am J Neuroradiol 2012;33: 474-80

19. McDougall CG, Spetzler RF, Zabramski JM, et al. The Barrow ruptured aneurysm trial. J Neurosurg 2012;116:135-44

20. Berthelsen CL. Evaluation of coding data quality of the HCUP National Inpatient Sample. Top Health Inf Manage 2000;21:10-23

21. Molyneux AJ, Kerr R, Langham J, et al. Applicability of coiling for subarachnoid haemorrhage. Lancet 2005;366:1924

22. Molyneux AJ, Kerr RS, Birks J, et al. Risk of recurrent subarachnoid haemorrhage, death, or dependence and standardised mortality ratios after clipping or coiling of an intracranial aneurysm in the international subarachnoid aneurysm trial (ISAT): long-term followup. Lancet Neurol 2009;8:427-33

23. Johnston SC, Dowd CF, Higashida RT, et al. Predictors of rehemorrhage after treatment of ruptured intracranial aneurysms: the cerebral aneurysm rerupture after treatment (CARAT) study. Stroke 2008;39:120-25 